\title{
Accelerated 4D flow imaging using randomly undersampled echo planer imaging with compressed-sensing reconstruction
}

\author{
Tamer A Basha*, Kraig V Kissinger, Beth Goddu, Sophie Berg, Reza Nezafat \\ From 17th Annual SCMR Scientific Sessions \\ New Orleans, LA, USA. 16-19 January 2014
}

\section{Background}

4D flow imaging using phase contrast CMR (PC CMR) allows visualization and quantification of blood flow. One of the major limitations of 4D flow imaging is its long scan time (in the order of 10-20 min). In this study, we sought to investigate an accelerate 4D flow imaging sequence that combines an efficient data sampling strategy using echo planar imaging (EPI) with randomly undersampled 3D k-space sampling pattern. The randomly undersampled k-space data are then reconstructed using compressed sensing (CS).

\section{Methods}

Figure 1 shows the k-space acquisition strategy. Similar to regular EPI acquisition, the $\mathrm{k}$-space data are divided into multiple segments. For each segment, the profiles are undersampled with the CS rate such that all EPI segments have the same undersampling pattern. The proposed EPI random sampling strategy was implemented. Seven subjects were recruited $(26 \pm 12$ years; 3 males) for 4D flow CMR on a $1.5 \mathrm{~T}$ Philips Achieva magnet. Images were acquired axially using a GRE sequence $\left(\mathrm{FOV}=340 \times 280 \times 60 \mathrm{~mm}^{3}\right.$, resolution $=2 \times$ $2 \times 3 \mathrm{~mm}^{3}, \mathrm{TR} / \mathrm{TE} / \alpha=7.4 / 3.8 \mathrm{~ms} / 20^{\circ}$, EPI factor $=3$, Turbo Factor $=2$, CS rate $=3$ ) in a volume covering the ascending and descending aorta, and the aortic bifurcation. Only foot-head flow encoding was used to provide an adequate temporal resolution of $30 \mathrm{~ms}$ for the measurements. A single beam navigator placed on the right hemi-diaphragm was used to gate the acquisition with the respiratory cycle. The nominal scan time for this scan was 3:30 minutes at $70 \mathrm{bpm}$ assuming $100 \%$ gating efficiency (vs. 8:40 minutes if standard parallel imaging with rate 4 was used). For each subject, the $4 \mathrm{D}-\mathrm{PC}$ scan was followed by a standard breath-hold 2D-PC scan with the same flow encoding direction $(\mathrm{FOV}=$ $340 \times 280 \mathrm{~mm} 2$, resolution $=2 \times 2 \mathrm{~mm}^{2}$, slice thickness $=5 \mathrm{~mm}^{3}$, SENSE rate $=2.5$ ). The acquired 2D slice is selected from the previously obtained $4 \mathrm{D}$ scan and approximately at the aortic bifurcation. Data are then transferred to a separate station where the CS reconstruction was performed using a total variation minimization algorithm. Next, flow quantifications were performed on both the ascending and the descending aorta for all acquisitions and then compared between the $2 \mathrm{D}$ scans and the corresponding slices in the $4 \mathrm{D}$ scans.

\section{Results}

Figure 2a shows representative mean velocity profiles for the ascending aorta of one volunteer using the $2 \mathrm{D}$ and 3D-EPI-CS acquisition. Figure $2 \mathrm{~b}$ show the correlation between the mean velocity measurements from the $2 \mathrm{D}$ and the 3D-EPI-CS scans $\left(R^{2}=0.93\right)$.

\section{Conclusions}

A combination of EPI and randomly underampling kspace will substantially reduce the $4 \mathrm{D}$ flow scan time. Our initial results show no systematic difference between flow measurements between 2D PC CMR and 3D PC CMR.

\section{Funding}

Samsung Electronics. 

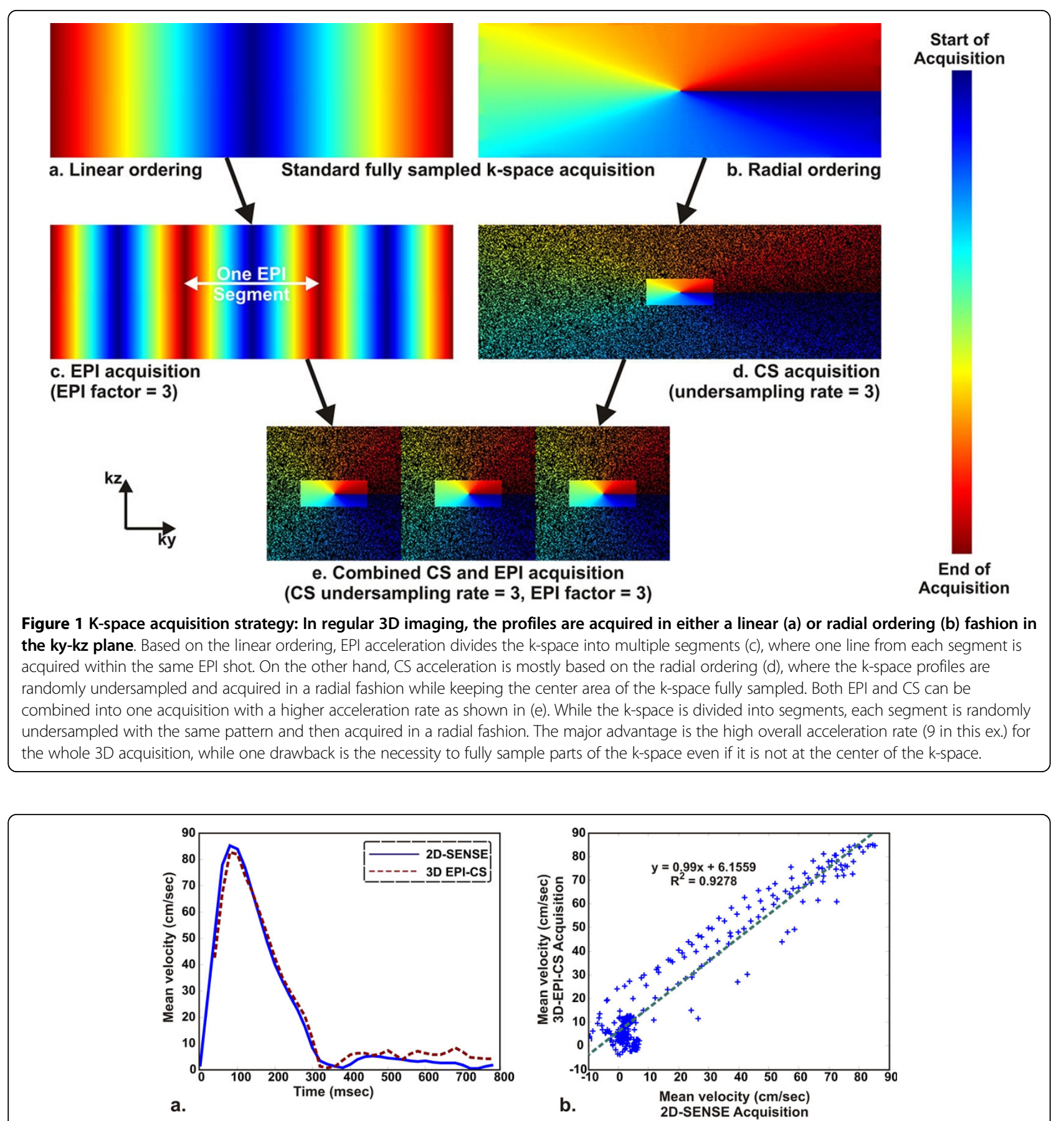

Figure 2 a) Example mean velocity of the blood flow in ascending aorta from one subject using the 2D acquisition and the proposed 3D acquisition. b) The correlation between the mean velocity measured from the 2D and the proposed 3D acquisitions. 